\title{
Análisis del Consumo de Coque de Petróleo en Algunos Sectores Industriales
}

\author{
Aldo R. Santos ${ }^{1}$ y Rogério J. Silva ${ }^{2}$ \\ (1) Universidad Santa Cecília, Departamento de Química, 11045-100 Santos, SP-Brasil \\ (e-mail: rsantos@unisanta.br) \\ (2) Universidad Federal de Itajubá, Instituto de Ingeniaría Mecánica, 37500-000 Itajubá, MG-Brasil \\ (e-mail: rogeriojs@unifei.edu.br)
}

\begin{abstract}
Resumen
Se analiza el consumo de coque de petróleo y el aumento de su producción en los últimos años. El procesamiento de los petróleos pesados genera menos fracciones leves y más fracciones pesadas, desequilibrando la matriz energética. Para retornar el equilibrio, la industria petrolífera opta por aumentar el número de unidades de craqueamiento, que consumen las fracciones pesadas y producen fracciones más livianas, generando como residuo el coque. Los diversos usos del coque generan contaminantes cuya emisión es regulada en distinta forma en distintos países. Se concluye que, cualquiera que sea la ruta a seguir para la utilización del coque de petróleo, los consumidores deberán considerar los patrones de emisión de contaminantes, principalmente de dióxido de azufre.
\end{abstract}

Palabras-clave: procesamiento de petróleo, coque de petróleo, sector industrial, contaminantes

\section{Consumption Analysis of Petroleum Coke in Some Industrial Segments}

\begin{abstract}
Petroleum coke consumption and the increase of its production during the recent years are analyzed. The processing of heavy oils produces less light fractions and more heavy fractions, unbalancing the energetic matrix. To balance this, the petroleum industry usually raises the number of coking units, which consumes the heavy fractions and produces lighter fractions, generating coke as residue. The different forms of using coke generate pollutants that are regulated and controlled in different forms by different countries. It is concluded that, independent of the form in which petroleum coke is consumed, the users should consider the amount of pollutants emitted, mainly sulfur dioxide.
\end{abstract}

Keywords: petroleum processing, petcoke, desulphurization, industrial sector, polluting 


\section{INTRODUCCIÓN}

El coque de petróleo es un subproducto de la industria de refinación de petróleo, el cual tiene alto poder calorífico y bajo precio. Debido a las cantidades cada vez mayores de petróleos pesados procesados, la producción de coque ha ido en aumento. La alta disponibilidad del coque de petróleo tornó este residuo atractivo para el sector industrial, principalmente para el sector de generación de energía eléctrica (Wang et al., 2004).

En la medida en que los petróleos son más pesados, sus densidades en grado API van disminuyendo y su contenido de contaminantes, principalmente los compuestos sulfurados, tienden a aumentar. Esta tendencia queda evidenciada en los datos presentados en la Fig. 1, que consta de un estudio hecho en el petróleo procesado, en los EUA de cerca de 14.8 millones de barriles/día; el cual actualmente, dos tercios son importados de varias partes del mundo (EIA, 2007a).

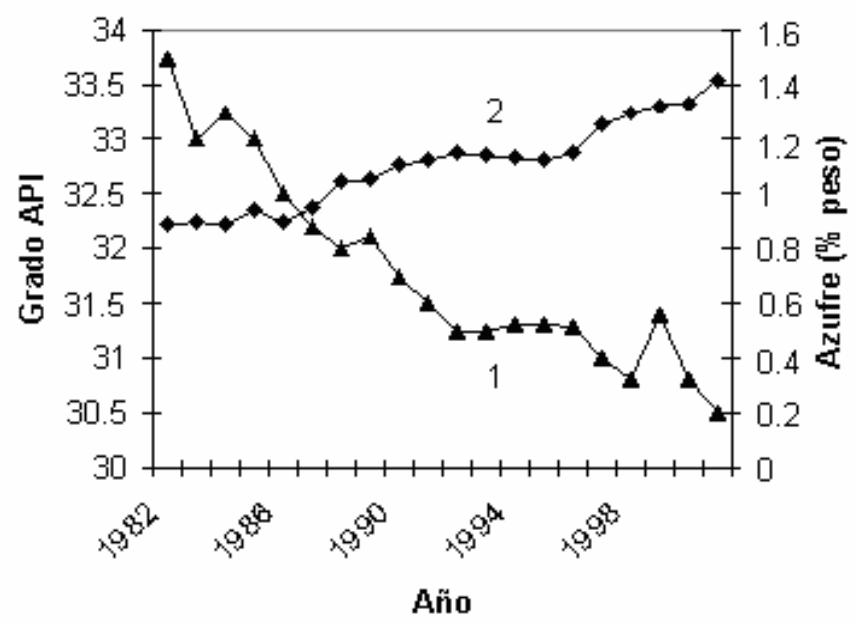

Fig.1: Evolución del grado API (1) y contenido de azufre (2) de las cargas procesadas por las refinerías de los EUA.

Debido a la necesidad de producción de las fracciones más ligeras del petróleo, como naftas, gasolina, queroseno y diesel; las refinerías de petróleo fueron adaptándose, procesando petróleo más pesado para atender al mercado consumidor de productos ligeros y medianos.

Así, junto con el craqueamiento catalítico fluido, que convierte gasóleos en gases y gasolina, el coquizado retardado fue tomando forma y ganando cada vez más importancia en las refinerías (ANP, 2007).

Como el coque de petróleo es uno de los productos originarios del fondo del barril en el sistema de procesamiento de petróleo, este derivado tiene mayor o menor contenido de azufre en función del tipo de petróleo el cual se origina. Así se pueden tener coques con altos y bajos contenidos de azufre (Salvador et al., 2003; Barros et al., 2003).

En este trabajo, se pretende mostrar que los petróleos ofertados en el mercado internacional actual son más pesados, y por lo tanto, presentan mayor contenido de contaminantes, producen menos fracciones ligeras y generan mas fracciones pesadas de consumo limitado. Para consumir esas fracciones pesadas y generar más fracciones ligeras, los refinadores optaran por instalar más unidades de craqueamiento, que atienden a esas dos necesidades, pero generan coque de petróleo, que concentra en si los contaminantes del petróleo, limitando su uso industrial. La industria hormigonera, debido las condiciones operacionales, surge como un consumidor de alto potencial.

\section{TIPOS DE PROCESOS DE PRODUCCIÓN DE COQUE DE PETRÓLEO}

Según Speight (2004), la producción de coque puede ser hecha por medio de tres procesos distintos: el coquizado retardado, el coquizado en lecho fluidizado y el coquizado en lecho fluidizado, con gasificación. 
El coque originado de las fracciones más pesadas del petróleo, concentra las impurezas más densas, como metales y compuestos sulfurosos; el contenido de esos componentes depende directamente de la calidad del petróleo procesado. Así, se pueden tener coques en el mercado internacional con contenidos de azufre que varíen entre de 4.0 a $7.5 \%$ en masa (Salvador et al., 2003). Los tres procesos de producción de coque de petróleo presentan diferentes configuraciones operacionales y de proceso, los cuales interfieren en la composición final del coque (Speight, 2004). En la Tabla 1 presenta la composición de los coques producidos por los tres procesos, utilizando carga tradicional de residuo de vacío, pero originadas de petróleos diferentes (Furimsky, 2000; Salvador et al., 2003; Garcia, 2002). La tabla solamente muestra la relación H/C, ligada a las condiciones del proceso, puesto que las cargas no tienen el mismo origen.

Tabla 1: Composición típica comparativa entre los tres procesos de coquizado.

\begin{tabular}{|c|c|c|c|}
\hline \multirow{2}{*}{$\begin{array}{c}\text { Composición } \\
\text { (\% en masa) }\end{array}$} & \multicolumn{3}{|c|}{ Proceso de Producción de Coque } \\
\cline { 2 - 4 } & Retardado & Lecho fluidizado & $\begin{array}{c}\text { Lecho fluido con } \\
\text { gasificación }\end{array}$ \\
\hline $\mathrm{C}$ & 87.9 & 86.3 & 94.9 \\
\hline $\mathrm{H}$ & 3.51 & 2.2 & 0.3 \\
\hline $\mathrm{H} / \mathrm{C}$ & 0.47 & 0.31 & 0.04 \\
\hline $\mathrm{N}$ & 1.61 & 2.4 & 1.1 \\
\hline $\mathrm{S}$ & 7.5 & 6.9 & 2.8 \\
\hline $\mathrm{O}$ & - & 0.9 & 0 \\
\hline Cenizas & 0.33 & 1.3 & 1.0 \\
\hline $\mathrm{d}\left(\mathrm{g} / \mathrm{cm}^{3}\right)$ & 2.00 & 0.80 & 0.96 \\
\hline
\end{tabular}

\section{PRODUCCIÓN MUNDIAL DE COQUE DE PETRÓLEO}

La producción mundial de coque de petróleo alcanzó los 81 Mt (millones de toneladas) en 2001, 83 Mt en 2002 (Dynamis, 2004) y llegando a 89 Mt en 2005 (EIA, 2007b). Estados Unidos de América es el mayor productor de coque de petróleo, con casi el $66 \%$ de la producción mundial. Cerca de $57 \%$ de la producción americana viene de la costa del Golfo de México (Texas y Louisiana), cerca de 35 refinerías producen coque de petróleo en cantidades apreciables (más de 1000 t/día). La Tabla 2 muestra la producción mundial de coque, considerando la participación porcentual por región productora de coque de petróleo (Dynamis, 2004).

Tabla 2: Producción mundial de coque de petróleo.

\begin{tabular}{|l|c|}
\hline Regiones productoras & Participación (\%) \\
\hline Norteamérica & 69.5 \\
\hline Sudamérica & 9.1 \\
\hline Europa & 8.5 \\
\hline Asia & 6.9 \\
\hline Ex-URSS & 5.0 \\
\hline África & 0.5 \\
\hline Oceanía & 0.5 \\
\hline
\end{tabular}

Entre los países exportadores de coque de petróleo según Energy Publishing (2007), en los meses de enero y febrero de 2007, los Estados Unidos exportarán 2628882 y 1919634 toneladas respectivamente, valores que corresponden al $93.28 \%$ y $91.7 \%$ del total de las exportaciones al nivel mundial; el segundo país colocado en el listado de exportadores fue China, con 132821 y 131597 tonelada, representando 4.71 y $6.28 \%$ en los mismos meses.

\section{PRECIO DEL COQUE DE PETRÓLEO}

Varios factores influencian el precio de mercado del coque de petróleo. Cuando el precio internacional del carbón aumenta, la demanda por coque de petróleo también aumenta. En la visión de los analistas, el alza del precio internacional del carbón generalmente es lo que más influencia el alza del precio del coque de petróleo. 
La Fig. 2 presenta la evolución, en US\$/millón de BTU, del costo del coque de petróleo en comparación del costo del gas natural y del gas sintético producido por la gasificación del coque de petróleo (GCPA, 2005).

La Tabla 3 presenta una comparación de precios (relativa a marzo de 2007) entre los coques producidos en la región del Golfo de los EUA y los producidos en Venezuela. En esta tabla, se observa que el contenido de azufre interfiere más en los precios que el HGI (Índice de Trituración Hardgrove) y que este tiene una tendencia alza de los precios a excepción de los producidos Costa Oeste de los EUA (Energy Publishing, 2007).

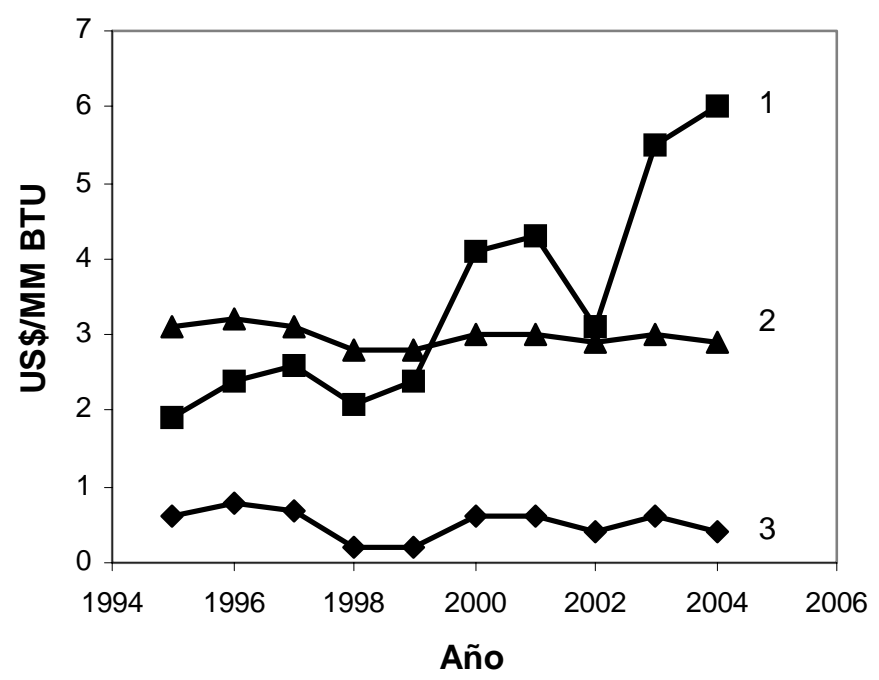

Fig.: 2 Evolución del precio del coque de petróleo (3), del gas natural (1) y del gas sintético derivado del coque de petróleo (2) (GCPA, 2005).

Tabla 3: Precios del coque de petróleo en función del contenido de azufre y dureza HGI.

\begin{tabular}{|c|c|c|c|c|}
\hline Origen & Azufre (\%) & $\mathrm{HGI}$ & Precio (US\$/t) & Tendencia \\
\hline $\begin{array}{c}\text { Estados Unidos (Región del Golfo) y } \\
\text { Venezuela }\end{array}$ & $4-5$ & $<50$ & 60.00 a 65.00 & Estable \\
\cline { 2 - 5 } & 6 & 35 a 45 & 50.00 a 52.00 & Crecer \\
\cline { 2 - 5 } & 6 & 50 a 70 & 53.00 a 56.00 & Crecer \\
\hline \multirow{2}{*}{$\begin{array}{c}\text { Estados Unidos } \\
\text { (Costa Oeste) }\end{array}$} & 3 & 45 a 50 & 60.00 a 63.00 & Estable \\
\cline { 2 - 5 } & $4^{+}$ & 45 a 50 & 57.00 a 61.00 & Crecer \\
\hline
\end{tabular}

Tabla 4: Comparación relativa entre los precios del carbón y del coque de petróleo.

\begin{tabular}{|c|c|c|}
\hline \multirow{2}{*}{ Intervalo (años) } & \multicolumn{2}{|c|}{ U\$ 0,01/MM BTU } \\
\cline { 2 - 3 } & Carbón & Coque de petróleo \\
\hline ene 97 a dic 97 & 120 & 89 \\
\hline dic 97 a nov 98 & 120 & 75 \\
\hline nov 98 a oct 99 & 118 & 68 \\
\hline oct 99 a sep 00 & 110 & 58 \\
\hline sep 00 a ago 01 & 108 & 70 \\
\hline ago 01 a jul 02 & 120 & 70 \\
\hline jul 02 a jun 03 & 118 & 52 \\
\hline jun 03 a may 04 & 120 & 70 \\
\hline may 04 a abr 05 & 135 & 110 \\
\hline abr 05 a may 06 & 155 & 140 \\
\hline may 06 a feb 07 & 160 & 165 \\
\hline
\end{tabular}

La Tabla 4 presenta una comparación relativa entre los precios de coque de petróleo y del carbón (Energy Publishing, 2007). Se aprecia que el precio del coque de petróleo siempre estuvo por debajo 
del precio del carbón; no obstante, a partir de julio de 2004, la tasa de crecimiento de los precios del coque de petróleo llevó a este a valores cada vez más próximos del precio del carbón. En julio de 2006 y enero de 2007 el precio del coque de petróleo superó el precio del carbón; por eso, la selección entre el uso de carbón o del coque de petróleo es técnica y no económica.

Por otro lado, la cantidad de coque de petróleo que una refinería produce tiene poco o nada que ver con el mercado. De hecho, las refinerías no deciden producir más coque de petróleo cuando el mercado crece o el precio sube. La única razón para que la producción de coque de petróleo aumente es cuando mayores cantidades de fracciones pesadas de petróleo son convertidas en fracciones mas ligeras, como combustible de aviación, que generan más residuos. En contrapartida, si una refinería está produciendo más fracciones pesadas, como aceite combustible, el proceso produce menos coque de petróleo.

\section{COQUE VERDE DE PETRÓLEO (CVP) Y COQUE DE PETRÓLEO CALCINADO (CPC)}

El coque obtenido directamente del proceso de coquizado retardado, es llamado de coque verde, debido al mayor contenido de componentes volátiles. En otra unidad operacional, el coque verde es sometido a un proceso llamado de calcinación, formando el llamado coque calcinado, cuyo contenido de volátiles es bastante reducido.

En el proceso de calcinación del coque verde, los calores sensible y latente de la materia volátil son aprovechados en una caldera recuperadora de calor, que genera vapor de agua a nivel de presión adecuado para la utilización en otras unidades en la propia empresa o para ser vendido a empresas próximas a la unidad de calcinación de coque verde. Así, la producción de vapor de agua puede ser considerada como un material de consumo, obtenido indirectamente, de la producción de coque de petróleo.

La Tabla 5 presenta la composición comparativa entre el coque verde y el coque calcinado grado ánodo, producido en Brasil por PETROCOQUE en Cubatão, São Paulo, originado de una mezcla de petróleos con bajo contenido de azufre (Hammond et al. 2005). En esta tabla se observa una gran diferencia entre el contenido de materia volátil en el coque verde y en el coque calcinado. También muestra que el $\mathrm{HGI}$ del coque calcinado es mucho menor que el $\mathrm{HGI}$ del coque verde. Como el $\mathrm{HGI}$ es inversamente proporcional a la viabilidad de pulverización del coque, se concluye que el coque verde presenta mejores condiciones de ser pulverizado que el coque calcinado, lo que indica que el coque verde es mucho más interesante para ser usado como combustible en relación al coque calcinado, pues se gasta menos energía para moler.

El mercado global de coque calcinado es altamente competitivo, participando varios países como: Canadá, Argentina, Brasil, Reino Unido, Alemania, Noruega, España, Sudáfrica, India, Indonesia, Japón, China y Rusia (Ellis y Paul, 2000).

Tabla 5: Composición comparativa entre el coque verde y calcinado grado ánodo.

\begin{tabular}{|c|c|c|}
\hline Especificación & $\begin{array}{c}\text { Coque } \\
\text { verde }\end{array}$ & $\begin{array}{c}\text { Coque } \\
\text { calcinado } \\
\text { (grado } \\
\text { ánodo) }\end{array}$ \\
\hline Materia volátil (\%) & 9.0 a 10.5 & 0.08 a 0.15 \\
\hline Humedad (\%) & 8.0 a 14 & 0.2 a 0.4 \\
\hline Cenizas (\%) & 0.09 a 0.14 & 0.10 a 0.18 \\
\hline Azufre (\%) & 0.70 a 0.85 & 0.70 a 0.78 \\
\hline Vanadio (ppm) & 180 a 230 & 200 a 250 \\
\hline Níquel $(p p m)$ & 180 a 200 & 200 a 220 \\
\hline Silica (ppm) & 40 a 80 & 20 a 60 \\
\hline Hierro (ppm) & 80 a 120 & 60 a 100 \\
\hline Sodio (ppm) & 70 a 90 & 50 a 100 \\
\hline Calcio (ppm) & 20 a 40 & 20 a 40 \\
\hline HGl & 70 a 80 & 20 a 40 \\
\hline
\end{tabular}




\section{PROCESOS QUE UTILIZAN COQUE DE PETRÓLEO}

Dependiendo de la oferta al mercado consumidor, hay casos en que el coque calcinado es usado como combustible en termoeléctricas y cementeras, a pesar del bajo contenido de volátiles (lo que dificulta la combustión) y del menor valor de HGI (indicando mayor dificultad en la pulverización del material). El coque verde puede tener los siguientes usos (Ellis y Paul, 1998):

1) coque combustible utilizado en la producción de cemento y en calderas de lecho fluidizado para la generación de vapor y energía eléctrica usando material calcáreo para la remoción de compuestos sulfurosos;

2) en caso de bajo contenido de azufre, el coque de petróleo puede tener uso metalúrgico en la forma de mezclas compuestas con coque mineral, para alimentación de alto-horno;

3) sufriendo oxidación parcial, el coque de petróleo puede ser usado en procesos de gasificación, para aplicación en la producción de vapor de agua, generación de energía eléctrica y producción de cargas gaseosas para varias aplicaciones industriales.

El coque verde puede ser también utilizado directamente en lo alto-horno, proceso conocido como inyección de finos de carbón. En este proceso el coque verde, también utilizado como combustible, es mezclado con otros carbones, pulverizado e inyectado directamente en los altos-hornos. Estas aplicaciones están siendo utilizadas con éxito en Brasil, en la COSIPA - Compañía Siderúrgica Paulista, en Cubatão, SP, haciendo que el sector siderúrgico sea un consumidor más de coque de petróleo (BR-N $\left.{ }^{0} 20,2005\right)$.

El coque calcinado, a su vez, tiene diversas aplicaciones industriales: en la fabricación de ánodos en la industria de aluminio, en la producción de titanio a partir del óxido de titanio, en la producción de monóxido de carbono para la producción de materiales plásticos y en la utilización en procesos industriales que requieran bajos contenidos de azufre, como en la producción de aceros especiales y acero inoxidable.

En la Fig. 3 se muestran los porcentajes del mercado consumidor de coque de petróleo, donde se aprecia que el sector cementero es el mayor consumidor de coque de petróleo, con $40 \%$ del total de coque producido. El segmento de calcinación de coque verde es el segundo mayor consumidor, con $22 \%$ de participación; el sector de plantas termoeléctricas se presentan como el cuarto consumidor de coque de petróleo, consumiendo 14\% del total de coque producido en 1999.

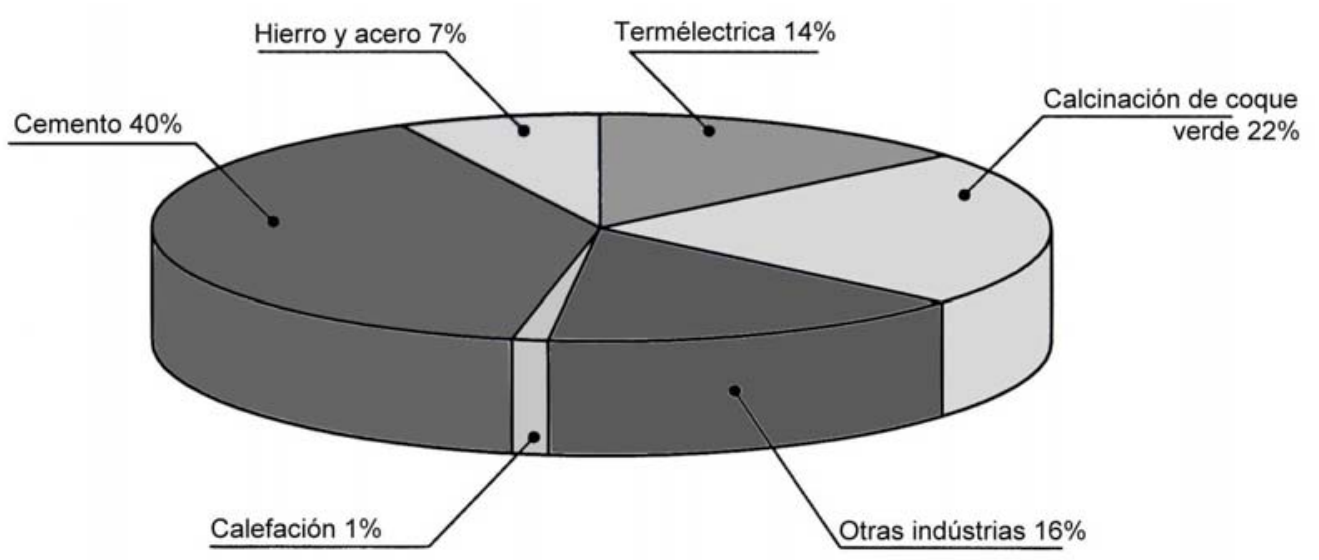

Fig. 3: Mercado consumidor de coque de petróleo, en 1999 (IEA-COAL, 2004).

El Consejo Mundial de Energía recomienda que la mezcla carbón mineral y coque de petróleo contenga un máximo de $20 \%$ de coque, debido al bajo contenido de material volátil en el coque de petróleo (WEC, 2005).

\section{NUEVAS TECNOLOGÍAS PARA EL CONSUMO DE COQUE DE PETRÓLEO}

Según Furimsky (1999), la oferta de petróleos pesados a ido aumentando en el mercado mundial. El procesamiento de esos petróleos genera menos productos ligeros y más residuos pesados. Así, 
habrá una tendencia de los refinadores para la utilización de procesos de gasificación de residuos en las propias refinerías de origen, produciendo corrientes gaseosas que servirán de materia-prima para otros procesos.

Wang et al (2004), propusieron un proceso con cero emisiones de $\mathrm{SO}_{2}$ y $\mathrm{CO}_{2}$ y, con aplicación prevista para gasificación del coque de petróleo. El coque es quemado en un lecho fluidizado, usando $\mathrm{CaO}$ para absorber $\mathrm{SO}_{2}$ y $\mathrm{CO}_{2}$. Un calcinador, quemando coque, podrá ser usado para regenerar el $\mathrm{CaO}$ y obtener $\mathrm{CO}_{2}$ para otros procesos industriales. La perdida del CaO será debida a su transformación en $\mathrm{CaSO}_{4}$, obligando a reposición de $\mathrm{CaCO}_{3}$ a través del calcinador.

\section{LEGISLACIÓN AMBIENTAL}

Debido a los contenidos de azufre, normalmente presentes en gran parte del coque de petróleo disponible en el mercado internacional, este trabajo únicamente se preocupa por los límites de emisión de $\mathrm{SO}_{2}$, pues este contaminante es producido por la quema del coque de petróleo.

En los EUA, el patrón de emisión para el $\mathrm{SO}_{2}$ establecido por la EPA para hornos de producción de clínquer de cemento depende de la localización, condiciones de dispersión atmosférica y proximidad con núcleos poblacionales.

En la Unión Europea, los patrones establecidos varían de país a país, pero son medidos en las condiciones de $25{ }^{\circ} \mathrm{C}, 101.3 \mathrm{kPa}$ y $10 \%$ de $\mathrm{O}_{2}$ libre en los gases de combustión en base seca. En estas condiciones, se considera que, para emisiones por debajo de $1000 \mathrm{mg} \mathrm{SO} / \mathrm{Nm}^{3}$, se recomienda tratamiento de los gases utilizando material calcáreo y, para emisiones por encima de $1000 \mathrm{mg} \mathrm{SO}_{2} / \mathrm{Nm}^{3}$, se recomienda utilizar sistemas de lavadores secos o húmedos. Para los hornos de producción de clínquer es recomendado mantener la emisión entre 170 a $340 \mathrm{mg} \mathrm{SO} / \mathrm{Nm}^{3}$ (UBA, 2001).

\section{LEGISLACIÓN AMBIENTAL EN BRASIL}

La Resolución CONAMA $\mathrm{n}^{0}$ 8, de 06 de Diciembre de 1990, que está en proceso de revisión, establece que para fuentes fijas con potencia menor de $70 \mathrm{MW}$ el límite de emisión es $5000 \mathrm{~g}$ $\mathrm{SO}_{2} / \mathrm{Mkcal}$ (carbón u aceite combustible). Para fuentes fijas con potencia mayor de $70 \mathrm{MW}$, el límite de emisión es $2000 \mathrm{~g} \mathrm{SO}_{2} / \mathrm{Mkcal}$ (carbón u aceite combustible).

La Resolución CONAMA $n^{0}$ 382, de 26 de Diciembre de 2006 establece los límites máximos de emisión de contaminantes atmosféricos para fuentes fijas. En su Artículo $7^{\circ}$, párrafo $1^{\circ}$, cita que el órgano medioambiental evaluador podrá establecer valores menos restrictivos que los limites máximos de emisión establecidos en esta Resolución, considerando las limitaciones tecnológicas y el impacto en las condiciones locales, de acuerdo con lo dispuesto en la Resolución CONAMA $n^{\circ} 5$ de 15 de Junio de 1989. En su Artículo $8^{\circ}$ establece que permanezcan aplicables los criterios y límites establecidos en la Resolución CONAMA $n^{\circ}$ 8, de 06 de Diciembre de 1990 para los procesos de generación de calor no establecidos por esta Resolución. La Resolución CONAMA $n^{\circ} 382$, en el ANEXO XI - Límites de emisión para contaminación atmosférica proveniente de la industria del cemento Portland, no establece limite de emisión para los óxidos de azufre $\left(\mathrm{SO}_{\mathrm{x}}\right)$, expuesta en el apartado 7 que "en función de las características locales del área de influencia de la fuente de contaminación de la calidad del aire, el órgano medioambiental licenciador podrá establecer límites de emisión más restrictivos, inclusive considerando la alternativa de utilizar combustibles con menor potencial de contaminación".

En el Estado de São Paulo, el padrón de emisión para la industria cementera es de $350 \mathrm{mg} \mathrm{SO} / \mathrm{Nm}^{3}$ a $7 \%$ de $\mathrm{O}_{2}$ libre en los gases de combustión, en base seca (Busato, 2004).

En el Estado de Paraná, la Resolución $\mathrm{n}^{0} 054$ de 2006 establece los límites de emisión de $\mathrm{SO}_{2}$, de acuerdo con la Tabla 6 (SEMA-PR, 2007). Para hornos de producción de clínquer, la Resolución establece el límite de $400 \mathrm{mg} \mathrm{SO} / \mathrm{Nm}^{3}$, con $7 \%$ de $\mathrm{O}_{2}$ libre en los gases en base seca. 
Tabla 6: Límites de emisión de $\mathrm{SO}_{2}$, para 7\% de $\mathrm{O}_{2}$ libre en los gases de exhaustión en base seca.

\begin{tabular}{|c|c|}
\hline Potencia Térmica (MW) & $\mathrm{mg} \mathrm{SO}_{2} / \mathrm{Nm}^{3}$ \\
\hline$<10$ & - \\
\hline $10-50$ & 3000 \\
\hline $50-100$ & 1400 \\
\hline$>100$ & 400 \\
\hline
\end{tabular}

\section{CONCLUSIONES}

El aumento de la producción de coque de petróleo es una consecuencia natural de la disminución del grado API de los petróleos disponibles actualmente en el mercado internacional. Esta tendencia queda reflejada con el hallazgo de grandes reservorios de petróleos "no convencionales", extremadamente pesados y con alto contenido de azufre. De este modo, el actual mercado consumidor de coque de petróleo debe ser ampliado, estimulando a los actuales consumidores a aumentar sus límites de uso, incluyendo nuevas tecnologías para el uso del coque de petróleo.

También debe ser tomado en cuenta la posibilidad de mezclas con carbones de bajo contenido de azufre, buscando alcanzar el importante mercado consumidor de las termoeléctricas. De este modo, podrá haber equilibrio entre a producción y el consumo de ese residuo del procesamiento del petróleo.

Sin embargo, cualquiera que sea la ruta a seguir para la utilización del coque de petróleo, los consumidores deberán considerar los patrones de emisión de contaminantes, principalmente en relación al $\mathrm{SO}_{2}$.

\section{REFERENCIAS}

ANP, Agência Nacional de Petróleo, Petróleo e Derivados, Refino de Petróleo e Processamento de Gás Natural, Esquemas de Produção, http://www.anp.gov.br/petro/refino_esquema_produção (2007).

Barros, F.C.C., W. Lázaro y C.N. Borges; Coking Considerations, Hydrocarbon Engineering: June, 61 $-65(2003)$

BR-N ${ }^{0}$ 20, Petrobras Distribuidora, PROASFALTO, 45, $\mathrm{N}^{\circ}$ 20, ano 4 Julho/Agosto 2005 BR, O Uso Pioneiro do Coque Verde, http://www.br.com.br/Portalbr/solucoes/SolucoesBR20.pdf (2005).

Busato, L.C.; Formação e Controle de Emissões em Fornos de Cimento, VI FIMAI - Feira Internacional de Meio Ambiente Industrial, Seminário de Co-processamento, novembro de 2004 , www.abcp.org.br/sala_de_imprensa/noticias/fimai/palestra12.pdf (2004)

CONAMA, Conselho Nacional do Meio Ambiente, Ministério do Meio Ambiente, Brasil, Resolução 8 de 06/12/1990, Dispõe Sobre Padrões de Qualidade do Ar, Previstos no PRONAR", http://www.mma.gov.br/port/conama/legiano.cfm?codlegitipo=3 (2007).

CONAMA, Conselho Nacional do Meio Ambiente, Ministério do Meio Ambiente, Brasil, Resolução 382 de 26/12/2006, Estabelece os Limites Máximos de Emissão de Poluentes para Fontes Fixas, ANEXO XI, http://www.mma.gov.br/port/conama/legiano.cfm?codlegitipo=3 (2007).

Dynamis, Dynamis Mecânica Aplicada Ltda, Coque de Petróleo: Parte 3 - Perspectivas, Disponibilidade, Preço, http://www.dynamismecanica.com.br/artigo005.php (2004).

EIA, Energy Information Administration, Weekly Petroleum Status Report, DOE/EIA-0208(2007-14), http://www.eia.doe.gov/pub/oil_gas/petroleum/data_publications/weekly_petroleum_status_report/curr ent/pdf/wpsrall.pdf (2007a).

EIA, Energy Information Administration, U.S. Refinery and Blender Net Production of Petroleum coke, Updated on 4/20/2007, http://www.tonto.eia.gov/dnav/pet/his/mckrpus1A.htm (2007b). 
Ellis, P. J. y C.A. Paul; Tutorial: Delayed Coking Fundamentals, Great Lakes Carbon Corporation, Topical Conference on Refinery Processing, Tutorial Session: Delayed Coking, Paper 29a (1998).

Ellis, P. J. y C.A. Paul; A. Tutorial: Petroleum Coke Calcining And Use of Calcined Petroleum Coke. Great Lakes Carbon Corporation, $3^{\text {rd }}$ International Conference on Refining Process, Session T9005 Tutorial: Delayed Coking (2000).

Energy Publishing, LLC Domestic and International, Petcoke Report, May 2007, Energy Publishing Petcoke FOB Price Index (Current settlement - Delivery during next 90 days) and Hill Daily Coal Index (2007).

Furimsky, E.; Gasification in Petroleum Refinery of $21^{\text {st }}$ Century, Oil \& Gas Science and Technology Rev. IFP: 54(5), 597-618 (1999).

Furimsky, E.; Characterization of cokes from Fluid/flexi-coking of heavy feeds, Fuel Processing Technology: 67, $205-230$ (2000).

Garcia, R.; Combustíveis e combustão industrial, Editora Interciência, Rio de Janeiro, Brasil (2002).

GCPA, Gulf Coast Power Association, April 14, 2005, ConocoPhillips, Petroleum Coke Gasification Synergies for Refineries, http://www.gulfcoastpower.org/default/S05-amick.pdf (2005).

Hammond, D.G. y otros seis autores; Review of fluid bed coking Technologies, Exxon Mobil Research and Engineering Comapy, PTQ AUTUMN 2003, http://www.prod.exxonmobil.com/ refiningtechnologies/pdf/Autumn03PTQFLKArticlepdfforweb.pdf (2005).

IEA-COAL, IEA Clean Coal Center, United Kingdom, http://www.ieacoal.org.uk/content/coal.org.uk/ content/default.asp?Pageld=604\&Languageld=0 (2004).

Revisão de 19 de setembro de 2005, Revisão da Resolução CONAMA 5, de 15/06/1989, do Ministério do Meio Ambiente, Brasil, Institui o Programa Nacional de Controle da Qualidade do Ar PRONAR, http://www.mma.gov.br/port/conama/processos/198FC8A8/Texto Base.pdf (2005).

Salvador, S., J.M. Commandré y B.R. Stanmore; Reaction rates for the oxidation of highty sulphurized petroleum cokes: the influence of thermogravimetric conditions and some coke properties, Fuel, 82, 715 - 720 (2003).

SEMA-PR, Secretaria de Meio Ambiente e Recursos Hídricos do Estado do Paraná, Brasil, Resolução no 054/06 - SEMA, Definição de Critérios para o Controle da Qualidade do Ar, www.pr.gov.br/meioambiente/pdf/res_054_06.pdf (2007).

Speight, J.G.; New Approaches to Hydroprocessing, Catalysis Today, 98 (1-2), 55 - 60 (2004).

UBA, Umwelt Bundes Amt, Cement and Lime Manufacturing Industries, Reference Document on Best Available Techniques in the Cement and Lime Manufacturing Industries, 23 - 24, December http://www.bvt.umweltbundesamt.de/archiv/G_Zement-u-Kalk.pdf (2001).

Wang, J., E.J. Anthony y J.C. Abanades; Clean and efficient use of petroleum coke for combustion and power generation: Fuel 83, 1341 - 1348 (2004).

WEC, World Energy Council; Challenges and Economics of Using Petroleum Coke for Power Generation, http://www.worldenergy.org/wec-geis/publications/default/tech_papers/17th_congress/ (2005) 
